\title{
Interaction dynamics of neuronal oscillations analysed using wavelet transforms
}

\author{
Xiaoli Li ${ }^{\mathrm{a}, *}$, Xin Yao ${ }^{\mathrm{a}}$, John Fox ${ }^{\mathrm{b}}$, John G. Jefferys ${ }^{\mathrm{b}}$ \\ ${ }^{a}$ Cercia, School of Computer Science, The University of Birmingham, Birmingham B15 2TT, UK \\ ${ }^{\mathrm{b}}$ Department of Neurophysiology, Division of Neuroscience, School of Medicine, The University of Birmingham, Birmingham B15 2TT, UK
}

Received 10 June 2006; received in revised form 10 August 2006; accepted 13 August 2006

\begin{abstract}
This paper describes the use of a computational tool based on the Morlet wavelet transform to investigate the interaction dynamics between oscillations generated by two anatomically distinct neuronal populations. The tool uses cross wavelet transform, coherence, bi-spectrum/bicoherence and phase synchronization.

Using specimen data recorded from the hippocampus of a rat with experimentally induced focal epilepsy, linear and non-linear correlations between neuronal oscillations in the CA1 and CA3 regions have been computed.

The results of this real case study show that the computational tool can successfully analyse and quantify the temporal interactions between neuronal oscillators and could be employed to investigate the mechanisms underlying epilepsy.
\end{abstract}

(C) 2006 Elsevier B.V. All rights reserved.

Keywords: Neuronal oscillation; Wavelet; Coherence; Synchronization; Bicoherence; Epileptic seizure

\section{Introduction}

Neural information processing depends not only on the firing rate of individual neurons, but also on the dynamic interplay among neurons within the local neuronal assembly and on the communication between different and often distant assemblies of neurons (Engel et al., 2001; Pesaran et al., 2002; Schnitzler and Gross, 2005). At the level of individual assemblies, neural activity often shows rhythmic synchronization with a frequency distribution, which varies according to the task being undertaken (e.g. perception and memory recall), neurophysiological state (e.g. sleep) or pathological activity (e.g. epilepsy) (Traub et al., 1999; Engel et al., 2001; Pesaran et al., 2002). It is also clear that the interaction between separate sites changes in different neurophysiological activities (Schnitzler and Gross, 2005; Leinekugel et al., 2002; Wolfgang et al., 1999), but at present, knowledge about the mechanisms underlying these interactions is limited.

Currently, measurements of coherence, phase delay and synchronization of neuronal oscillations are widely used when

\footnotetext{
* Corresponding author. Tel.: +44 1214145142.

E-mailaddresses: xiaoli.avh@gmail.com(X.Li),j.g.r.jefferys@bham.ac.uk (J.G. Jefferys).
}

investigating the organization and function of the neuronal networks during normal and abnormal cognitive processing. Correlation of EEG activity recorded from two different brain sites is often used to describe interaction dynamics (Leinekugel et al., 2002), but the methods are usually based on the assumption that the data is stationary; spectral coherence (Wolfgang et al., 1999), for example, is often used to estimate the correlation between Fourier transforms of two simultaneous EEG recordings. The main disadvantages of existing methods are that the Fourier spectrum is limited to stationary signals and temporal interactions are missed or ignored. In particular, the non-linear relations between two neural networks have a direct negative effect on the correlation estimated with traditional methods (Lachaux et al., 2002).

There is increasing interest in the use of wavelet-based techniques in processing non-stationary EEG recordings, not only with respect to oscillatory behaviour (Klein et al., 2006; Bullock et al., 1997; Li et al., 2005a,b), but also for spike detection (Senhadji and Wendling, 2002; Latka et al., 2003; Nenadic and Burdick, 2005), sleep stage identification (Jobert et al., 1994; Kiymik et al., 2004), anesthetics (Kochs et al., 2001) and filtering (Glassman, 2005). In addition to providing spectral statistics similar to those obtained with Fourier analysis, wavelet-based methods are capable of detecting 
the short lived temporal interactions, which occur in EEG recordings.

In this paper, a computational tool with Morlet wavelet transform (MWT) is used to detect the temporal interactions between two anatomically distinct neuronal populations. Cross wavelet transforms, coherence, bicoherence and phase synchronization are produced and the performance of the computational tool is confirmed using a real case study. The results show that linear and non-linear correlations between neural oscillations at the CA1 and CA 3 regions in the hippocampus can be detected. We suggest that this is a potential tool for studying the mechanism of neural information processing and epileptic seizures.

\section{Methods}

Continuous wavelet transform (CWT) can decompose a signal into a set of finite basis functions. The decay of wavelet function makes CWT able to uncover transient characteristics hidden in the EEG series. Wavelet coefficients $\mathrm{W}(s, \tau)$ are produced through the convolution of a parent wavelet function $\psi(t)$ with the analysed signal $x(t)$; it is (Meyer, 1993):

$W(s, \tau)=\frac{1}{\sqrt{s}} \int x(t) \psi^{*}\left(\frac{t-\tau}{s}\right) \mathrm{d} t$,

where $s$ and $\tau$ denote the scale and translation and $*$ denotes complex conjugation. By adjusting the scale $s$, a series of different frequency components in the signal can be extracted. The factor $\sqrt{s}$ is for energy normalization across the different scales. Through wavelet transforms, the information of the time series $x(t)$ is projected on the two dimension space (scale $s$ and translation $\tau$ ).

In this study, Morlet wavelet is employed; it is given by:

$\psi_{0}(t)=\pi^{-1 / 4} \mathrm{e}^{i \omega_{0} t} \mathrm{e}^{-1 / 2 t^{2}}$

where $\omega_{0}$ is the wavelet central angle frequency, often $\omega_{0} \geq 6$, which is an optimal value to adjust the time-frequency resolution (Farge, 1992). Morlet wavelet is a Gaussian-windowed complex sinusoid; the Gaussian's second order exponential decay of Morlet function gives a good time localization at the time domain (Torrence and Compo, 1998). Another reason to choose Morlet wavelet is that the complex Morlet wavelet transform (MWT) can obtain the amplitude and phase of neural activity simultaneously. Therefore, MWT is able to investigate the coherence/synchronization between neural activities simultaneously recorded at two different sites. The complex Paul wavelet transform could be also employed to estimate amplitude and phase, but the Paul function is more sharply defined in time and is therefore better suited for detecting pulse-like variations than for analysing neural activity.

Given an EEG time series, $\mathrm{X}=\left\{x_{n}\right\}, n=0, \ldots, N-1$, with equal time spacing $\mathrm{d} t$, the continuous wavelet transform of the discrete sequence is defined as the convolution of $x_{n}$ with a scaled and translated version of $\psi_{0}(t)$; it is given by

$W(s, \tau)=\sum_{n^{\prime}=0}^{N-1} x_{n^{\prime}} \psi^{*}\left(\frac{\left(n^{\prime}-\tau\right) \mathrm{d} t}{s}\right)$ where $*$ denotes the complex conjugate. Changing the wavelet scale $s$ and translating along the localized time index $\tau$, a map can be constructed to show the amplitude of any feature versus the scale at a short time. Large values of the map (wavelet coefficients) reflect the combined effect of a large fluctuation of the time series and of a good matching of shape between the series and the wavelet function.

Based on MWT, the wavelet power of a time series X at the time scale space is called as scalogram; it is defined as

$W_{\mathrm{X}}=|W(s, \tau)|^{2}$.

Scalogram describes the variance of this series at the time scale plane. To depict the covariance between two series, a cross wavelet power is defined. Given a pair of time series, $\mathrm{X}$ and $\mathrm{Y}$, with their MWT denoted $W_{X}$ and $W_{Y}$, a cross wavelet power may be defined to identify correlation between the two time series using the product of their wavelet coefficients; the product is defined as follows:

$W_{\mathrm{XY}}(s, \tau)=W_{\mathrm{X}}(s, \tau) W_{\mathrm{Y}}^{*}(s, \tau)$,

where $*$ means complex conjugation. The plot of $\left|W_{\mathrm{XY}}\right|^{2}$ is called a coscalogram, which displays the coincident events of two series at the time scale plane.

In order to better describe the phase and amplitude correlations from two scalograms, two new measures are proposed to indicate the essentially intermittent dynamics in two different series, instead of the direct method of coscalogram. First, the wavelet local correlation coefficient (WLCC) (Buresti and Lombardi, 1999), it is defined as follows:

$\operatorname{WLCC}(s, \tau)=\frac{\mathrm{R}\left[W_{\mathrm{XY}}(s, \tau)\right]}{\left|W_{\mathrm{X}}(s, \tau)\right|\left|W_{\mathrm{Y}}(s, \tau)\right|}$,

where $\mathrm{R}$ means the real part of $W_{\mathrm{XY}}$. WLCC is a measure of the phase correlation between two series at the time-scale domain. A second measure is the cross wavelet coherence function (CWCF) (Sello and Bellazzini, 2000) which is defined as follows:

$\operatorname{CWCF}(s, \tau)=\frac{2\left|W_{\mathrm{XY}}(s, \tau)\right|^{2}}{\left|W_{\mathrm{X}}(s, \tau)\right|^{4}+\left|W_{\mathrm{Y}}(s, \tau)\right|^{4}}$.

The CWCF is a measure of the amplitude correlation of two series at the time-scale domain, which ranges from 0 (no correlation) to 1 (strong correlation).

To refine the relationship between two neural activities, wavelet coherence is proposed to quantify the first order relationship. Wavelet coherence derives from the form of the Fourier based coherence function, namely the ratio of the cross spectrum to the product of the auto-spectrum of the two series $\mathrm{X}(t)$ and $\mathrm{Y}(t)$. Wavelet coherence is given by

$\left(c^{W}(s, \tau)\right)^{2}=\frac{\left|S_{\mathrm{XY}}^{w}(s, \tau)\right|^{2}}{S_{\mathrm{XX}}^{w}(s, \tau) S_{\mathrm{YY}}^{w}(s, \tau)}$,

Herein, the localized power spectrum above is

$S_{\mathrm{XY}}^{w}(s, \tau)=\frac{1}{s \int_{T} W_{\mathrm{X}}(s, \tau) W_{\mathrm{Y}}^{*}(s, \tau) d \tau}, T=[\tau-\Delta \tau, \tau+\Delta \tau]$. 
where $T$ is selected based on the time resolution desired in the coherence map. Auto-spectrum $S_{\mathrm{XX}}^{w}(s, \tau)$ and $S_{\mathrm{YY}}^{w}(s, \tau)$ can be done by Eq. (8b). The integration in Eq. (8b) effectively provides an ensemble averaging localized in time and reduces the variance from noise (8). At the same time, the phase difference of wavelet coherency can be calculated by cross-spectrum; it is given by

$\phi(s, \tau)=\tan ^{-1}\left(\frac{\mathrm{R}\left(S_{\mathrm{XY}}^{w}(s, \tau)\right)}{\mathrm{I}\left(S_{\mathrm{XY}}^{w}(s, \tau)\right)}\right)$

where $\mathrm{R}$ and $\mathrm{I}$ are the real and imaginary parts of the cross spectrum. The phase difference is a function of time and scale.

Although the coscalogram, WLCC, CWCF and wavelet coherence can reveal an intermittent linear correlation between the two series, non-linear interactions such as phase coupling between the two time series cannot be revealed by these methods. It has been proved that bicoherence spectrum is a powerful tool to detect the phase coupling among series. Bicoherence based on Fourier transform (FT) has been applied to quantify phase coupling between a pair of EEG recordings (Bullock et al., 1997); this is based on the principle that the sum and difference frequencies in the non-linear system are generated with a fixed phase relationship. Calculation of the bicoherence enables us to quantify the amount of phase coupling and number of independent signal sources. Unfortunately, Fourier-based bicoherence cannot detect short-term non-linear interactions. To overcome this disadvantage of the Fourier method, a wavelet-based bicoherence has been introduced (van Milligen et al., 1995). Similar to Fourier-based method, a time-scale wavelet cross-bispectrum (van Milligen et al., 1995) is defined by

$B_{\mathrm{XXY}}^{W}\left(s_{1}, s_{2}\right)=\int_{T} W_{\mathrm{X}}\left(s_{1}, \tau\right) W_{\mathrm{X}}\left(s_{2}, \tau\right) W_{\mathrm{Y}}(s, \tau) \mathrm{d} \tau$

where $1 / s=\left(1 / s_{1}\right) \pm\left(1 / s_{2}\right)$; this relation is the same as the frequency relation $f=f_{1} \pm f_{2}$. The calculation of the wavelet bicoherence is below:

$\left(b_{\mathrm{XXY}}^{W}\left(s_{1}, s_{2}\right)\right)^{2}=\frac{\left|B_{\mathrm{XXY}}^{W}\left(s_{1}, s_{2}\right)\right|^{2}}{\int_{T}\left|W_{\mathrm{X}}\left(s_{1}, \tau\right) W_{\mathrm{X}}\left(s_{2}, \tau\right)\right|^{2} \mathrm{~d} \tau \int_{T}\left|W_{\mathrm{Y}}(s, \tau)\right|^{2} \mathrm{~d} \tau}$

Wavelet bicoherence ranges from 0 to 1 ; when there is no coupling, the value is 0 ; when two series are phase coupled absolutely, it is 1. Advantages of wavelet bicoherence are that it can detect phase coupling over short time intervals and it can identify zero bicoherence for two series with no phase coupling; this differs from results using Fourier methods (simulations can be found in Chung and Powers, 1998).

Coherence and bicoherence can successfully analyse the linear and non-linear correlations of EEG activity recorded at two different sites, however, coherence and bicoherence depend on amplitude and phase in the time series. The phase relationship may be identified independently by measuring phase synchronization. Based on cross spectrum Eq. (8b), the instantaneous phase $\phi(t)$ and instantaneous amplitude $A(t)$ of series $x(t)$ can be obtained; $\phi(t)$ and $A(t)$ only have a clear physical meaning if $x(t)$ is a narrow-band signal (Rosenblum et al., 2001). If two periodic oscillators $\left(\phi_{1}, \phi_{2}\right)$ are weakly coupled, the relation of their phase dynamics is given by

$$
\begin{aligned}
& \frac{\mathrm{d} \phi_{1}(t)}{\mathrm{d} t}=w_{1}+\varepsilon g_{1}\left(\phi_{1}, \phi_{2}\right) \\
& \frac{\mathrm{d} \phi_{2}(t)}{\mathrm{d} t}=w_{2}+\varepsilon g_{2}\left(\phi_{2}, \phi_{1}\right)
\end{aligned}
$$

where $w_{1}$ and $w_{2}$ are the frequencies of two oscillators and $g_{1}$ and $g_{2}$ are the coupling terms which are 2-pi period in both arguments, and $\varepsilon$ is the coupling coefficient. The interaction between the two oscillators essentially affects the evolution of their phases if the frequency $w_{1}$ and $w_{2}$ are in resonance: $n w_{1} \approx$ $m w_{2}$ (Rosenblum et al., 2001). The phase difference of two series is given by

$\varphi_{n, m}(t)=n \phi_{1}(t)-m \phi_{2}(t)$

Sometimes synchronization can be detected in a straightforward way by analysing the generalized phase difference $\varphi_{\mathrm{n}, \mathrm{m}}(t)$. The phase difference plot can trace transitions between synchronous and non-synchronous states that are due to non-stationarities in interacting systems and or coupling. In general, the distribution of the cyclic relative phase $\psi_{\mathrm{n}, \mathrm{m}}(t)=\varphi_{\mathrm{n}, \mathrm{m}}(t) \bmod 2 \pi$ is analysed to describe phase synchronization; the presence of a peak in the distribution of $\psi_{\mathrm{n}, \mathrm{m}}(t)$ indicates the presence of some interaction. In order to trace the variation of the strength of interaction between two neural activities, an intensity of the first Fourier mode of the distribution is given by:

$$
\gamma_{n, m}(t)=\sqrt{\left\langle\cos \left(\psi_{n, m}(t)\right)\right\rangle^{2}+\left\langle\sin \left(\psi_{n, m}(t)\right)\right\rangle^{2}}
$$

where brackets denote the average over time. The measure of synchronization strength varies from 0 to 1 , called the synchronization index. To simplify the computation, this paper only considers $n=m$. The details of phase synchronization can be found in Rosenblum et al. (2001) and Mormann et al. (2000).

When applying the above methods to neural activities, the significance level or confidence limits need to be; this is because (a) patterns of neural activity vary with time and (b) data collection, in different experiments, may not be made under identical conditions. Since there is no reliable theoretical formula to estimate the significance level, a statistical method based on surrogate techniques, also called as resampling, must be employed. This gives confidence levels and enables acceptance/rejection of a null hypothesis. A disadvantage of re-sampling is that it needs long computation time. The basic procedure of the surrogate technique is given below:

(a) given two neural activities $\mathrm{X}$ and $\mathrm{Y}$, a statistic of interest is computed through the wavelet transform; for instance, wavelet coherence: $\theta=\mathrm{WT}(\mathrm{X}, \mathrm{Y})$;

(b) resample $\mathrm{Y}$ through a statistic method such as bootstrap by $N$ times; a surrogate dataset is obtained, $\mathrm{Y}^{i}(i=1, \ldots, N)$;

(c) for each surrogate set $i$, the statistic of interest can be computed, for example wavelet coherence $\theta^{i}=\mathrm{WT}\left(\mathrm{X}, \mathrm{Y}^{i}\right)$;

(d) from the tails of the $\theta^{i}$ distribution, a confidence limit for the statistic of interest may be obtained. 


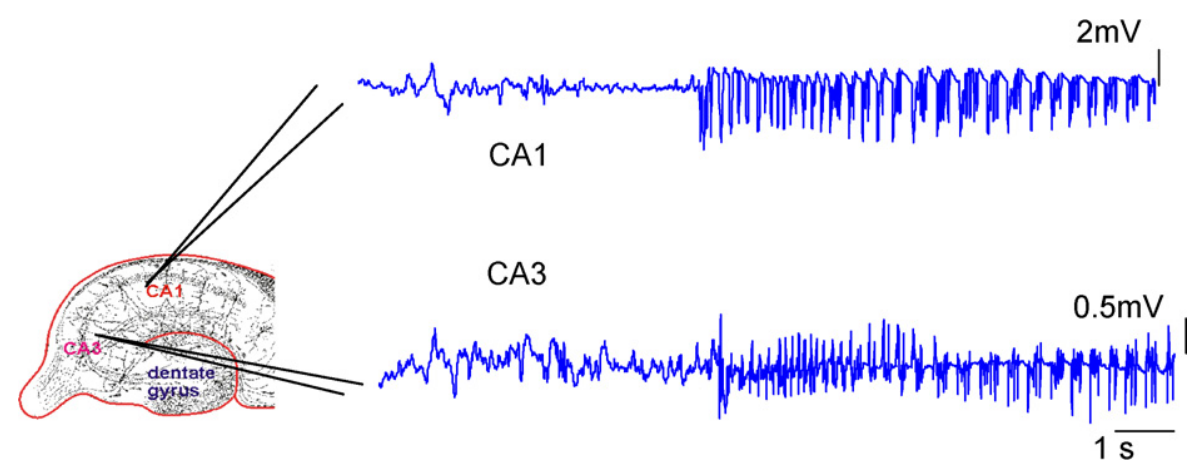

Fig. 1. Neuronal activity recorded from the CA1 and CA3 regions of the hippocampus during the transition from pre-seizure to seizure states (the duration of this recording is $12 \mathrm{~s}$, the sampling frequency is $2500 \mathrm{~Hz}$ ).

In the above procedure, the core is to select a suitable resampling method for the statistic of interest; different statistics of interest need different resampling methods. The more details can be found in Kantz and Schreiber (2003), the program on this technique can be obtained at their website. In general, an intuitive and simple way is a bootstrap. Bootstraps may be applied, for example, when estimating the amplitude relation of two neural activities (e.g. CWCF). When investigating the phase relation of two neural activities, however, non-linear resampling should be considered. A typical method is amplitude adjusted fourier transform (AAFT) (Schreiber and Schmitz, 1996). Another improved method is the iterative AAFT (IAAFT) generator (Theiler et al., 1992). The AAFT is applied in the present study and the procedure is outlined below. The original series is denoted by the
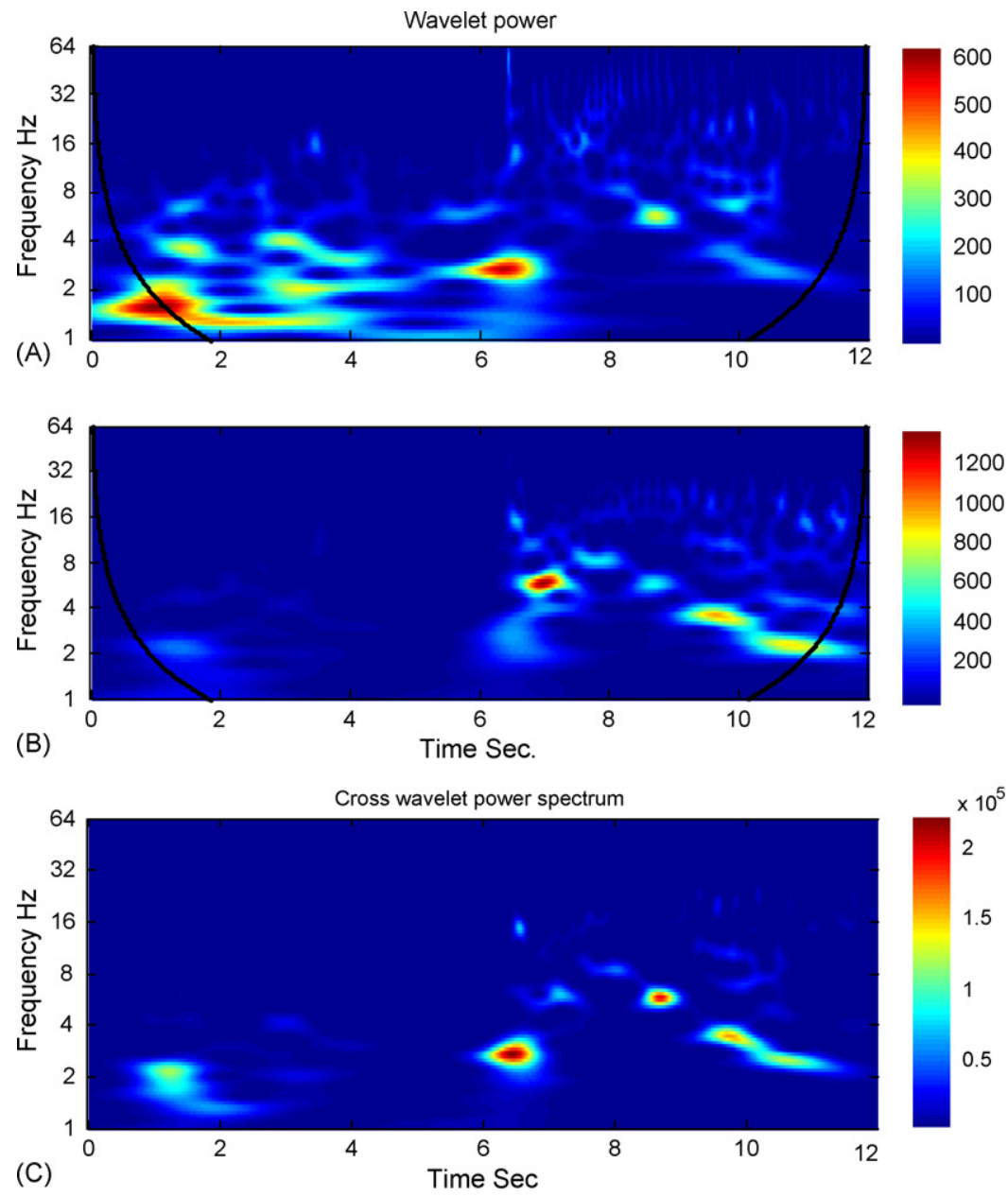

Fig. 2. Scalogram and coscalogram of the neuronal oscillations in Fig. 1 with Morlet wavelet transform. (A) The wavelet power of EEG recording in the CA1. (B) The wavelet power of EEG recording in the CA3. (C) Coscalogram of two Neuronal oscillations in the CA3 and CA1. The bright highlight indicates the same oscillations occurring at the same time. The black curve line indicates the 'cone of influence'. (For interpretation of the references to color in this figure legend, the reader is referred to the web version of the article.) 
vector $\{y(n)\}$. First, the Fourier spectrum of the vector $\{y(n)\}$ is calculated. Then, a random shuffle is applied to adjust the amplitudes of spectrum. To preserve power spectrum at the surrogated series, the squared coefficients of the surrogated series are replaced by those of the original time series. At the same time, the phases in the Fourier spectrum are randomized. Finally, an inverse Fourier transform is applied to construct a new series. The new series will have the same power spectrum as the original series, but the phase is different. The non-linear resampling method is suitable for estimating the confidence level for wavelet coherence, bicoherence and phase synchronization because these estimations depend on phase relationships. For example, this method may be applied to estimate the confidence level of bicoherence between two EEG recordings. Assume that there is a fixed phase relation between the gamma wave $(40 \mathrm{~Hz})$ in X EEG series and the beta wave $(20 \mathrm{~Hz})$ in Y EEG series; the above surrogate techniques can generate a new series $\mathrm{Z}$, similar to the Y EEG series, but with different phase. Since the phase relation of $\mathrm{X}$ series and $\mathrm{Z}$ series is not fixed, the bicoherence of these two series should be reduced to close to zero. However, the estimated bicoherence contains the influence of others factor such as data size, data lengths and so on. Thus, many surrogated $\mathrm{Z}$ series can be used to estimate the confidence level of the $\mathrm{X}$ and Y series through simple statistics.

\subsection{Data recordings}

The recordings used in this study were obtained from earlier investigations into the rat tetanus toxin model of focal epilepsy (Finnerty and Jefferys, 2000, 2002). Methods were described in the previous reports, but briefly, male Sprague-Dawley rats were anaesthetised with halothane, bipolar recording electrodes were placed into CA1 and CA3 of both hippocampi, and 4-5 ng of tetanus toxin (Wellcome Foundation Research Laboratories, Beckenham, Kent, UK) was injected. The animals were allowed to recover with free access to food and water and recording started 3-6 days postoperatively. The data were sampled at $2.5 \mathrm{kHz}$ (the experiments were carried out in accordance with the Animal Scientific Procedures Act 1986).

In this study, the Morlet wavelet transform tool was used to analyse the data shown in Fig. 1. The neuronal oscillations show a progression from pre-ictal to ictal states, the ictal event beginning $5 \mathrm{~s}$ after the onset of the trace. The analysis which follows will focus on the relationship between neural activity in the CA1 and CA3 regions of the hippocampus, with a view to identifying the interaction dynamics of two regions. There is anatomical evidence of a projection from CA3 to CA1 (Li et al., 1994).

\section{Results}

Wavelet transforms can be used to analyse non-stationary signals (van Milligen et al., 1995; Li et al., 2005a,b) and localized wavelet coefficients are well suited for analysing EEG data because they can indicate transient and evolutionary phenomena hidden in the EEG data (Saab and Gotman, 2005; Lachaux et al., 1999; Li et al., 2005a,b). In the following section, the application of wavelet transform to the real EEG recording in Fig. 1 is described; the aim is to describe the interaction dynamics of two neural networks during an epileptic seizure.

The wavelet power of the EEG data in Fig. 1 is plotted in Fig. 2A and B. Outside of the black curve indicates the 'cone of influence', which means the region of wavelet spectrum due to
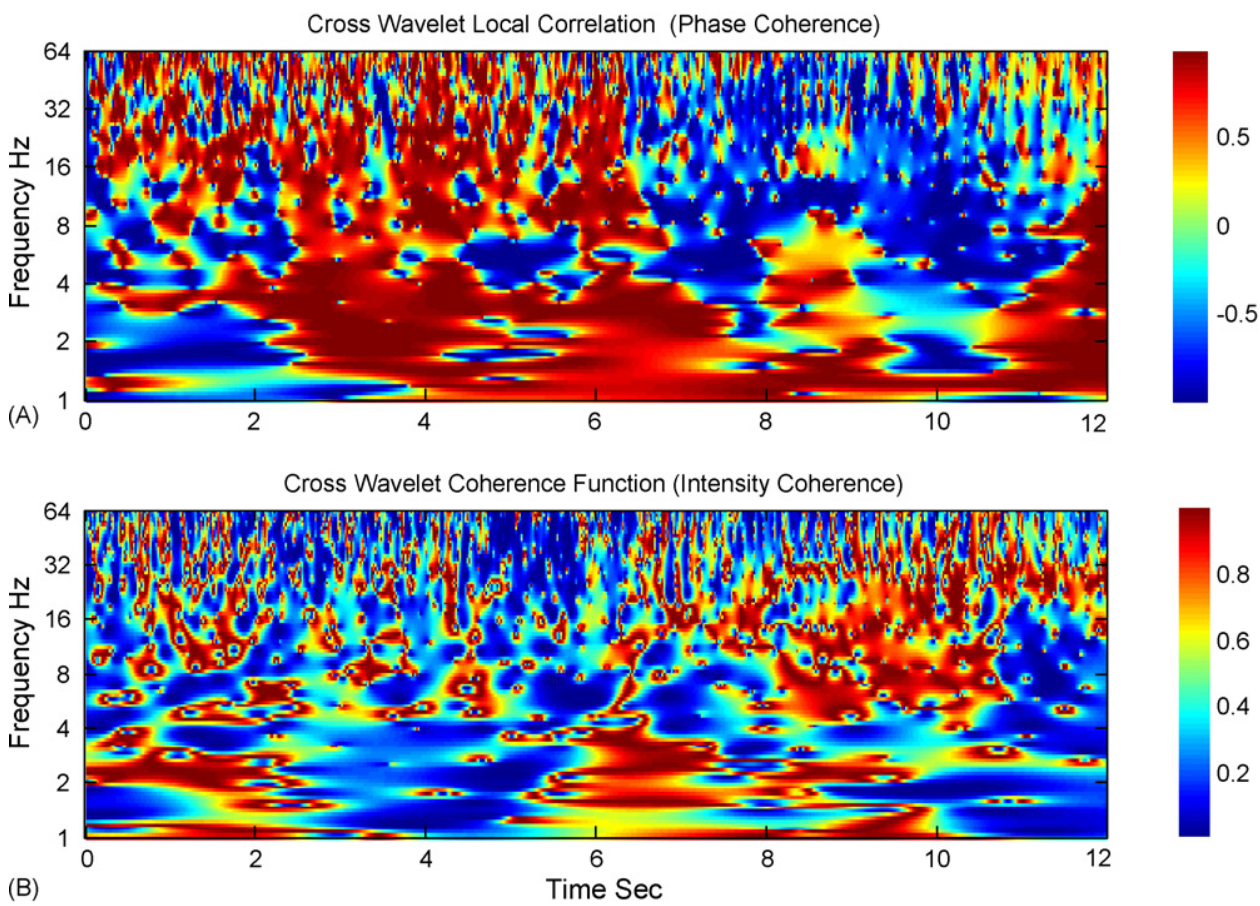

Fig. 3. (A) Wavelet local correlation, which is a measure of phase correlation between two signals at the time-frequency plane. (B) Cross wavelet coherence function, which is a measure of amplitude correlation between two signals at the time-frequency plane. 


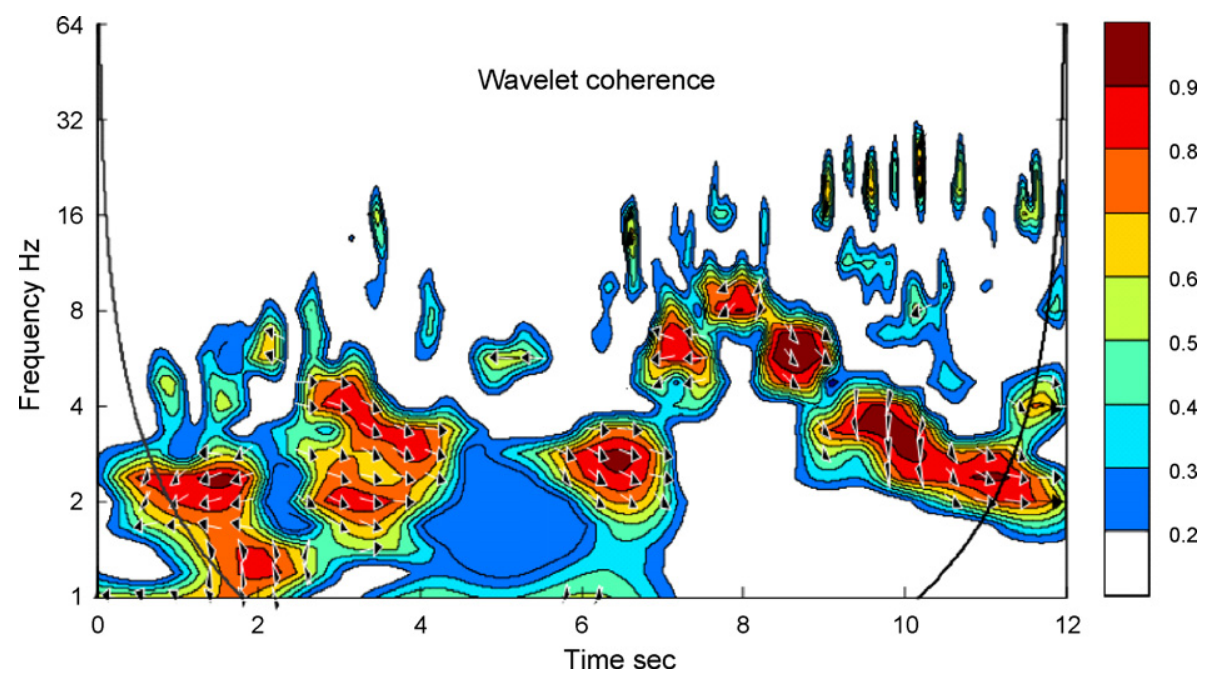

Fig. 4. Wavelet coherence of two neuronal oscillations from the CA3 and CA1 regions. The phase difference of cross spectrum is shown by arrow direction (in-phase: arrow pointing right, means CA3 leads CA1; anti-phase: arrow pointing left, means CA1 leads CA3). The amplitude and phase information in the EEG signal are integrated to describe the first-order relationship between the EEG signals. The thick black line nearby the warmest colour (red) is the significant level of $99 \%$ with re-sampling of AFFT. (For interpretation of the references to color in this figure legend, the reader is referred to the web version of the article.)

the edge effect. From the wavelet power of neuronal oscillations, the components that occur at the different time and frequency are indicated at the time-frequency domain. The difference between neuronal oscillations at the pre-ictal and ictal stage is clear; during the ictal stage there are more high frequency components. The scalogram displays the frequency content of the EEG data over time; in particular, the transients embedded in the EEG data can be revealed. As shown in Fig. 1, two EEG data sets are denoted as CA3 (denoted by X) and CA1 (denoted by Y), and their MWT are $W_{\mathrm{X}}$ and $W_{\mathrm{Y}}$, respectively The plot of $\left|W_{\mathrm{XY}}\right|^{2}$ displays the coincident events at the time-frequency domain in the two EEG data sets. Fig. $2 \mathrm{C}$ plots the coscalogram of the two neuronal oscillations in Fig. 1. The highlight points in the figure identify areas of correlation, which means that there is a correlation between neuronal oscillations in the CA3 and CA1 subfields at the same frequency band. In particular, it is easy to identify changes in correlation at the transition from preseizure to seizure state in Fig. 2C; this contrasts with the results from traditional correlation techniques, which do not demonstrate temporal correlation of different frequency components. Cross wavelet power is a measure of the similarity of power, at different frequency bands, in two separate neuronal oscillators.

Cross wavelet transform is a 'direct' measure of correlation of two neural activities at the time-frequency domain, so the values estimated contain bias and noise information, in addition this method does not reveal the relationship of amplitude and phase at two neural activities separately. Often, the phase of neural activity is more important than its amplitude during the neural signal processing. To this end, WLCC and CWCF may be used, and are plotted in Fig. 3 for the data given in Fig. 1. Fig. 3 A shows phase coherence of activity at the two neural sites; it is found that the phase coherence decreases during the transition

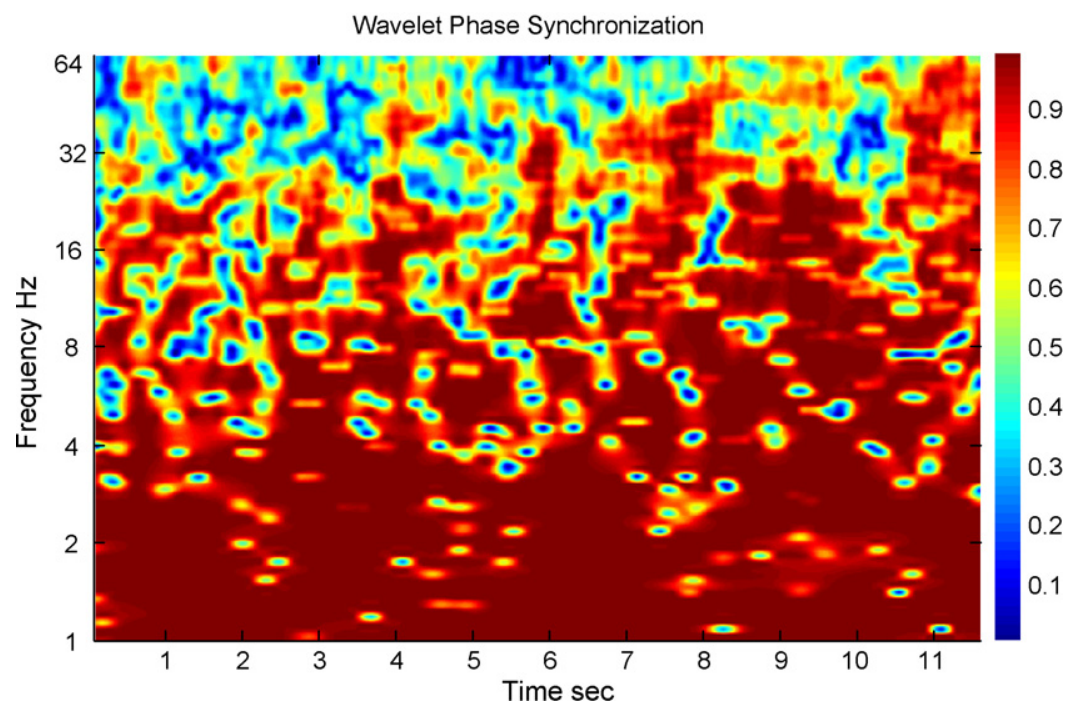

Fig. 5. The phase synchronization of neuronal oscillations at the CA3 and CA1. The bright colour indicates that there is phase synchronization in this area (time-frequency). (For interpretation of the references to color in this figure legend, the reader is referred to the web version of the article.) 
from interictal to ictal. However, Fig 3B shows that the intensity coherence increases during the transition from interictal to ictal. This difference may reflect different mechanisms of neural synchronization.

Wavelet coherence provides a view of the localized correlation with respect to both the time and frequency. Often, wavelet coherence is called the first order correlation between the two neural oscillations. Since the integration in wavelet coherence reduces the bias of correlation, wavelet coherence can give a more accurate localized correlation in the time-frequency domain. In Fig. 4, a wavelet coherence between the neuronal oscillations from CA1 and CA3 is plotted. It can be seen that, in the pre-ictal phase, there is a strong correlation in the $1-4 \mathrm{~Hz}$ frequency band. By contrast, in the first part of the ictal phase, the correlation is highest in the frequency band of $4-8 \mathrm{~Hz}$, falling to $2-4 \mathrm{~Hz}$ later in the ictal phase; in addition, correlations at the frequency band of $8-32 \mathrm{~Hz}$ can be seen during the ictal stage. In brief, wavelet coherence can accurately represent the covariance between two neuronal oscillations. The phase difference of the two neural oscillations is also shown as arrows in Fig. 4 through cross wavelet spectrum; horizontal arrows pointing from left to right signify in-phase signals, whilst horizontal arrows pointing from right to left indicate anti-phase. The phase difference of cross spectrum (arrow direction) is not identical to the wavelet coherence; at the area of strong wavelet coherence, in-phase or anti-phase is not found. This finding is similar to CWCL and CWCF in Fig. 3. Also, the wavelet coherence cannot completely describe the interaction of two neuronal oscillations, since it simultaneously contains the amplitude and phase information of neuronal oscillations.

Fig. 5 shows the phase synchronization between the neural oscillations recorded from CA1 and CA3. The width of the temporal window is set as $1 \mathrm{~s}$ with overlap of $50 \%$, which is a compromise between noise reduction and temporal resolution. It is found the strongest synchronization is in the frequency band of 1-4 Hz. These low frequency oscillators are coupled by phase. Comparing the pre-ictal and ictal stages, it is found that the high frequency oscillators are only coupled by phase during the ictal stage.

Above methods may reveal an intermittent linear correlation between two neural oscillations, the non-linear correlation such as phase coupling between two neural oscillations is not indicated; phase coupling can be estimated by bicoherence/bispectrum based on power spectrum (Bullock et al., 1997; Barnett et al., 1971; Jeffrey and Chamoun, 1994; Johansen and Sebel, 2000).

In the present analysis, wavelet bicoherence can reveal the higher order correlation and quantify non-linear relationship between the neuronal oscillations in the CA3 and CA1 regions. In Fig. 6, the bicoherence is plotted for the pre-ictal (A), seizure onset (B) and ictal (C) stages. It can be seen that, during the ictal stage, phase coupling between CA1 and CA3 is much more marked than during the pre-ictal phase. This change is especially remarkable at low oscillation and fast oscillation frequencies. During the pre-ictal stage, the phase coupling concentrates on the oscillations with similar frequency band. The phase coupling reduces at the stage of seizure onset. The modulation of
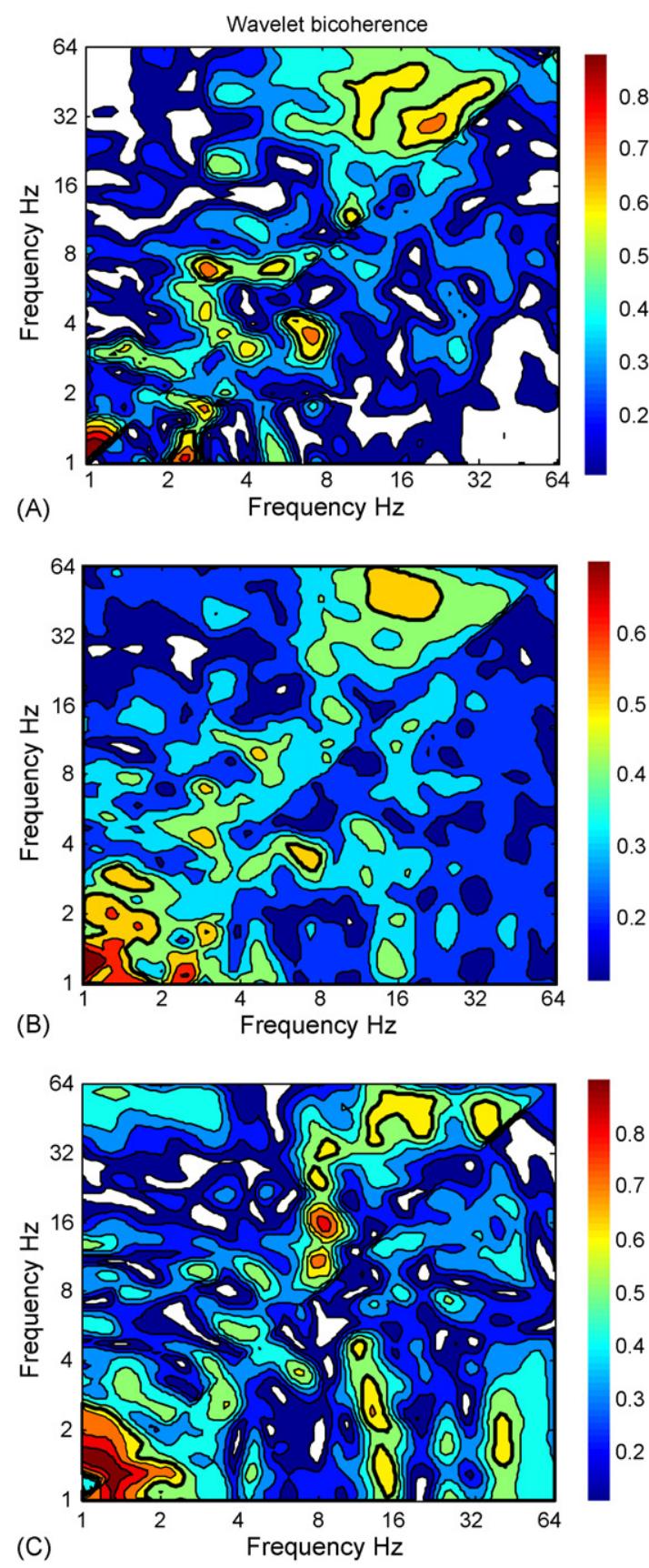

Fig. 6. Bicoherence of the neuronal oscillations from the CA 3 and $\mathrm{CA} 1$ regions at the pre-ictal stage, seizure start and ictal stage. The thick black lines on the contour plot are the significant level of $99 \%$ with re-sampling of AFFT. (For interpretation of the references to color in this figure legend, the reader is referred to the web version of the article.)

low oscillation and fast oscillation may mean that the fast oscillations are superimposed on the low frequency oscillation.

\section{Conclusion}

In this paper, a computational tool based on the Morlet wavelet transform is proposed to analyse two neural oscillations. The cross wavelet transform, coherence, bicoherence and phase synchronization of two neuronal oscillations has been described. 
The interaction dynamics between two neuronal oscillations were revealed through a real case. In the future work, this computational tool will be extended to analyse the multiple neural oscillations and further reveal a mechanism of synchronous network oscillation.

\section{Acknowledgements}

The work is supported by The Wellcome Trust, Advantage West Midlands (AWM), UK, and National Natural Science Foundation of China (60575012).

\section{References}

Barnett TP, Johnson LC, Naitoh P, Hicks N, Nute C. Bispectrum analysis of electroencephalogram signals during waking and sleeping. Science 1971;172:401-2.

Bullock TH, Achimowicz JZ, Duckrow RB, Spencer SS, Iragui-Madoz VJ. Bicoherence of intracranial EEG in sleep, wakefulness and seizures. Electroencephalogr Clin Neurophysiol 1997;103:661-78.

Buresti G, Lombardi G. Application of continuous wavelet transforms the analysis of experimental turbulent velocity signals. Proceedings of the first international symposium on turbulent shear flow phenomenon, S. Barbara, USA, September 1999.

Chung J, Powers EJ, The statistics of wavelet-based bicoherence., 1998. Proceedings of the IEEE-SP international symposium on time-frequency and time-scale analysis $1998 ; 141-4$.

Engel AK, Fries P, Singer W. Dynamic predictions: oscillations and synchrony in top-down processing. Nat Rev Neurosci 2001;2:704-16.

Farge M. Wavelet transforms and their applications to turbulence. Annu Rev Fluid Mech 1992;24:395-457.

Finnerty T, Jefferys JRG. 9-16 Hz oscillation precedes secondary generalization of seizures in the rat tetanus toxin model of epilepsy. J Neurophysiol 2000;83:2217-26.

Finnerty GT, Jefferys JRG. Investigation of the neuronal aggregate generating seizures in the rat tetanus toxin model of epilepsy. J Neurophysiol 2002;88:2919-27.

Glassman EL. A wavelet-like filter based on neuron action potentials for analysis of human scalp electroencephalographs. IEEE Trans Biomed Eng 2005;52:1851-62.

Jeffrey CS, Chamoun NG. An introduction to bispectral analysis for the electroencephalogram. J Clin Monit 1994;10:392-404.

Jobert M, Tismer C, Poiseau E, Schulz H. Wavelets-a new tool in sleep biosignal analysis. J Sleep Res 1994;3:223-32.

Johansen JW, Sebel P. Development and clinical application of electroencephalographic bispectrum monitoring. Anesthesiology 2000;93:1336-44.

Kantz H, Schreiber T. Nonlinear time series analysis. Cambridge University Press; 2003, http://www.mpipks-dresden.mpg.de/ tisean/.

Kiymik MK, Akin M, Subasi A. Automatic recognition of alertness level by using wavelet transform and artificial neural network. J Neurosci Methods 2004; 139:231-40

Klein A, Sauer T, Jedynak A, Skradies W. Conventional and wavelet coherence applied to sensory-evoked electrical brain activity. IEEE Trans Biomed Eng 2006;53:266-72.
Kochs E, Stockmanns G, Thornton C, Nahm W, Kalkman CJ. Wavelet analysis of middle latency auditory evoked responses: calculation of an index for detection of awareness during propofol administration. Anesthesiology 2001;95:1141-50.

Lachaux JP, Rodriguez E, Martinerie J, Varela FJ. Measuring phase synchrony in brain signals. Hum Brain Mapp 1999;8:194-208.

Lachaux JP, Rudrauf Lutz A, Cosmelli D, Quyen MLV, Martinerie J, Varela F. Estimating the time-course of coherence between single-trial brain signals: an introduction to wavelet coherence. Clin Neurophysiol 2002;32:157-74.

Latka M, Was Z, Kozik A, West BJ. Wavelet analysis of epileptic spikes. Phys Rev E 2003;67, 052902.

Leinekugel X, Khazipov R, Cannon R, Hirase H, Ben-Ari Y, Buzsáki G. Correlated bursts of activity in the neonatal hippocampus in vivo. Science 2002;296:2049-52.

Li X, Yao X, Jefferys JGR, Fox J, Computational neuronal oscillation with morlet wavelet transform. Proceedings of 27th annual international conference of the IEEE engineering in medicine and biology Society, Shanghai, SEP., IEEE Press, 1-4/2005.

Li X, Kapiris PG, Polygiannakis J, Eftaxias KA, Yao X. Fractal spectral analysis of pre-epileptic seizures phase: in terms of criticality. J Neural Eng 2005b;2:11-6.

Li XG, Somogyi P, Ylinen A, Buzsaki G. The hippocampal CA3 network: an in vivo intracellylar labeling study. J Comp Neurol 1994;339:108-81.

Meyer Y. Wavelets-algorithms and applications. S.I.A.M; 1993.

Mormann F, Lehnertz K, David P, Elger CE. Mean phase coherence as a measure for phase synchronization and its application to EEG of epilepsy patients. Physica D 2000;144:358-69.

Nenadic Z, Burdick JW. Spike detection using the continuous wavelet transform. IEEE Trans Biomed Eng 2005;52:74-87.

Pesaran B, Pezaris JS, Sahani M, Mitra PP, Andersen RA. Temporal structure in neuronal activity during working memory in macaque parietal cortex. Nat Neurosci 2002;5:805-11.

Rosenblum M, Pikovsky A, Kurths J, Schafer C, Tass PA. Phase synchronization: from theory to data analysis. Handbook of biological physics, Elsevier Science, Neuro-information and Neural modelling 2001; 279-321.

Saab ME, Gotman J. A system to detect the onset of epileptic seizures in scalp EEG. Clin Neurophysiol 2005;116:427-42.

Schnitzler A, Gross J. Normal and pathological oscillatory communication in the brain. Nat Rev Neurosci 2005;6:285-96.

Schreiber T, Schmitz A. Improved surrogate data for nonlinearity tests. Phys Rev Lett 1996;77:635-8.

Sello S, Bellazzini J, Wavelet cross-correlation analysis of turbulent mixing from large-eddy-simulations, Physics/0003029-arxiv.org, 2000.

Senhadji L, Wendling F. Epileptic transient detection: wavelets and time-frequency approaches. Clinical Neurophysiology 2002;32:175-92.

Theiler J, Eubank S, Longtin A, Galdrikian B, Farmer JD. Testing for nonlinearity in time series: the method of surrogate data. Physica D 1992;58:77-94.

Traub RD, Jefferys JGR, Whittington MA. Fast oscillations in cortical circuits. Cambridge, MA: MIT Press; 1999.

Torrence C, Compo GP. A practical guide to wavelet analysis. Bull Am Meteor Soc 1998;79:61-78.

van Milligen BP, Sánchez E, Estrada T, Hidalgo C, Brañas B, Carreras B, Garcia L. Wavelet bicoherence: a new turbulence analysis tool. Phys Plasmas 1995;2:3017-32.

Wolfgang HRM, Hristoph B, Matthias A, Herbert W, Edward T. Coherence of gamma-band EEG activity as a basis for associative learning. Nature 1999;397:434-6. 